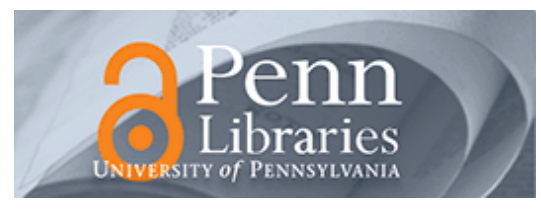

University of Pennsylvania

ScholarlyCommons

$5-1994$

\title{
Sums of Squares of Edge Lengths and Spacefilling Curve Heuristics for the Traveling Salesman Problem
}

Jun Gao

John M. Steele

University of Pennsylvania

Follow this and additional works at: https://repository.upenn.edu/oid_papers

Part of the Mathematics Commons, and the Science and Mathematics Education Commons

\section{Recommended Citation}

Gao, J., \& Steele, J. M. (1994). Sums of Squares of Edge Lengths and Spacefilling Curve Heuristics for the Traveling Salesman Problem. SIAM Journal on Discrete Mathematics, 7(2), 314-324. http://dx.doi.org/ $10.1137 /$ S0895480192240652

This paper is posted at ScholarlyCommons. https://repository.upenn.edu/oid_papers/256

For more information, please contact repository@pobox.upenn.edu. 


\title{
Sums of Squares of Edge Lengths and Spacefilling Curve Heuristics for the Traveling Salesman Problem
}

\author{
Abstract \\ The sum of squares of the edge lengths of the tour provided by the spacefilling curve heuristic applied to \\ a random sample of $n$ points from the unit square is proved to be asymptotically equal to a periodic \\ function of the logarithm of the sample size.

\section{Keywords} \\ spacefilling curves, traveling salesman problem (TSP), minimal spanning tree, combinatorial optimization, \\ order statistics, sums of squares

\section{Disciplines} \\ Mathematics | Science and Mathematics Education
}




\title{
SUMS OF SQUARES OF EDGE LENGTHS AND SPACEFILLING CURVE HEURISTICS FOR THE TRAVELING SALESMAN PROBLEM $^{*}$
}

\author{
JUN $\mathrm{GAO}^{\dagger}$ AND J. MICHAEL STEELE $\ddagger$
}

\begin{abstract}
The sum of squares of the edge lengths of the tour provided by the spacefilling curve heuristic applied to a random sample of $n$ points from the unit square is proved to be asymptotically equal to a periodic function of the logarithm of the sample size.
\end{abstract}

Key words. spacefilling curves, traveling salesman problem (TSP), minimal spanning tree, combinatorial optimization, order statistics, sums of squares

AMS subject classifications, primary 05C05, secondary $60 \mathrm{~F} 15$

1. Two sources of motivation. Two lines of investigation come together to form the motivation for the present work. The first of these concerns the behavior of the sums of squares of the edge lengths in several classical problems of geometric combinatorial optimization. The second concerns recent progress in the understanding of the behavior of the spacefilling curve heuristic for the traveling salesman problem (TSP).

Sums of squares of edges. This line begins with an empirical discovery of R. Bland. Since we obtain the same minimum spanning tree (MST) for a set of $n$ points $\left\{x_{1}, x_{2}, \ldots, x_{n}\right\} \subset[0,1]^{2}$ whether we assign edge costs $c_{i j}$ that are equal to the Euclidean length $\left\|x_{i}-x_{j}\right\|$ or to the square of the lengths $\left\|x_{i}-x_{j}\right\|^{2}$, we can save some computation time by working with the squared lengths. When Bland used the sum of squared edge lengths as the feature of merit in a study of algorithms for the MST, he found after computing the MST of a number of random samples of different sizes from $[0,1]^{2}$ that the value of the minimum value of the sum of squared edge lengths showed very little dependence on $n$ and little variation between samples. Bland was led to conjecture that there is a constant $C_{\text {MST }}$ such that for the MST of $\left\{X_{1}, X_{2}, \ldots, X_{n}\right\}$ where the $X_{i}$ are independent random variables with the uniform distribution on $[0,1]^{2}$, we have

$$
\lim _{n \rightarrow \infty} \sum_{e \in \mathrm{MST}}\|e\|^{2}=C_{\mathrm{MST}}
$$

with convergence in probability. This conjecture was proved in Aldous and Steele [1].

The method used to prove (1) relied on the possibility of calculating the MST via a greedy algorithm. Still, there are many functionals that are closely related to the MST for which there is no such possibility. Probably the most studied of these is the TSP that asks for the shortest tour through the points $\left\{x_{1}, x_{2}, \ldots, x_{n}\right\} \subset[0,1]^{2}$. We do not know at present whether the analogue to (1) holds for the TSP.

For the worst-case analysis, the state of knowledge for the TSP is more complete. Snyder and Steele [10] showed that there is a universal constant $C_{\text {TSP }}$ such that, for

* Received by the editors November 23, 1992; accepted for publication (in revised form) March 2, 1993.

$\dagger$ Portfolio Management International, Swiss Bank Corporation, 10 East 50th Street 32nd floor, New York, New York 10022.

$\$$ Department of Statistics, Wharton School, University of Pennsylvania, Philadelphia, Pennsylvania 19104 (steele@wharton.upenn.edu). This author's research was supported in part by National Science Foundation grant DMS92-11634 and Army Research Office grant DAAL03-91-G-0110. 
any $S=\left\{x_{1}, x_{2}, \ldots, x_{n}\right\} \subset[0,1]^{2}$ and any tour $T$ of $S$ of minimal length, we have

$$
\sum_{e \in T}\|e\|^{2} \leq C_{\mathrm{TSP}} \log n
$$

In subsequent analyses, Bern and Epstein [3] showed that the logarithmic term of (2) could not be replaced with a more slowly growing function.

Limit theory for the spacefilling heuristic. The spacefilling curve heuristic rests on the existence of a surjective mapping $\psi:[0,1] \rightarrow[0,1]^{2}$ such that for each $x \in[0,1]^{2}$ we can quickly compute a $t \in[0,1]$ such that $\psi(t)=x$. Formally, given $\left\{x_{1}, x_{2}, \ldots, x_{n}\right\} \subset[0,1]^{2}$, we have a three-step process where we (1) compute a set of points $\left\{t_{1}, t_{2}, \ldots, t_{n}\right\} \subset[0,1]$ such that $\psi\left(t_{i}\right)=x_{i}$ for each $1 \leq i \leq n$, (2) order the $t_{i}$ so that $t_{(1)} \leq t_{(2)} \leq \cdots \leq t_{(n)}$, and, finally, (3) define a permutation $\sigma:[1, n] \rightarrow[1, n]$ by requiring $x_{\sigma(i)}=\psi\left(t_{(i)}\right)$. The path that visits $\left\{x_{1}, x_{2}, \ldots, x_{n}\right\}$ in the order of $x_{\sigma(1)}, x_{\sigma(2)}, \ldots, x_{\sigma(n)}$ will be called the spacefilling curve path, and the tour that closes this path by adding the step from $x_{\sigma(n)}$ back to $x_{\sigma(1)}$ will be called the spacefilling curve tour.

Here we will focus on the behavior of the spacefilling curve heuristic in the context of the simplest possible stochastic model, where the points to be toured are modeled by independent random variables $X_{i}, 1 \leq i \leq n$ that are uniformly distributed in $[0,1]^{2}$. In [4], results of Platzman and Bartholdi [8] were refined to show that for a large class of spacefilling curves (SFCs) the length $L_{n}^{\mathrm{SFC}}=L^{\mathrm{SFC}}\left(X_{1}, X_{2}, \ldots, X_{n}\right)$ of a spacefilling heuristic tour through $\left\{X_{1}, X_{2}, \ldots, X_{n}\right\}$ satisfies

$$
\lim _{n \rightarrow \infty} \frac{E L_{n}^{\mathrm{SFC}}}{\sqrt{n} \varphi\left(\log _{p} n\right)}=1
$$

where $p$ is an integer depending on the geometry of the spacefilling curve and where $\varphi$ is a continuous periodic function of period 1 that is bounded away from zero. This behavior offers a novel contrast to that of the length $L_{n}^{\mathrm{OPT}}=L_{n}^{\mathrm{OPT}}\left(X_{1}, X_{2}, \ldots, X_{n}\right)$ of the shortest tour through the random sample $\left\{X_{1}, X_{2}, \ldots, X_{n}\right\}$, where the theorem of Beardwood, Halton, and Hammersley [2] declares that for the optimal solution no periodic term is needed; rather, there is simply a constant $\beta>0$ such that for $n \rightarrow \infty$ we have

$$
L_{n}^{\mathrm{OPT}} / \sqrt{n} \rightarrow \beta
$$

where the convergence takes place in expectation as well as with probability 1 .

The purpose of this article is to provide a precise asymptotic understanding of the sum of the squares of the edges of the tour provided by the spacefilling curve heuristic

$$
S_{n}=\sum_{i=1}^{n}\left\|X_{\sigma(i)}-X_{\sigma(i+1)}\right\|^{2}=\sum_{i=1}^{n}\left\|\psi\left(t_{(i)}\right)-\psi\left(t_{(i+1)}\right)\right\|^{2},
$$

where we invoke the convention that

$$
\sigma(n+1) \stackrel{\text { def }}{=} \sigma(1) \text { and } t_{(n+1)} \stackrel{\text { def }}{=} t_{(1)} .
$$

We will establish the possibly surprising fact that for a large class of spacefilling curves the value of this random variable is well approximated by a periodic function of the logarithm of the sample size. 
2. Main result. The first three properties described below are found in many classical spacefilling curves, including those of Hilbert [5] and Peano [7]. For these and related curves, the ordinary Lipschitz property and the bimeasure-preserving property are established in Milne [6]. The dilation and translation properties are easily verified by direct consideration of the traditional constructions, and only minor alterations of the usual constructions are needed to obtain curves with the circular Lipschitz property Assumption 4.

Assumption 1 (dilation property). There is an integer $p \geq 2$ such that, for all $0 \leq s, t \leq 1$,

$$
\|\psi(s)-\psi(t)\|=\sqrt{p}\left\|\psi\left(\frac{s}{p}\right)-\psi\left(\frac{t}{p}\right)\right\| .
$$

Assumption 2 (translation property). For $1 \leq i \leq p$, if $(i-1) / p \leq s, t \leq i / p$, then

$$
\|\psi(s)-\psi(t)\|=\|\psi(s+1 / p)-\psi(t+1 / p)\| .
$$

Assumption 3 (bimeasure-preserving property) Given any Borel set $A$ in $[0,1]$, the set $\psi(A)$ is measurable, and

$$
\lambda_{1}(A)=\lambda_{2}(\psi(A)),
$$

where $\lambda_{d}$ is the Lebesgue measure on $\mathbb{R}^{d}$.

Assumption 4 (circular Lipschitz property). There is a constant $c_{\psi}$ such that

$$
\|\psi(s)-\psi(t)\| \leq c_{\psi} \rho(s, t)^{1 / 2}
$$

where $\rho$ is the circular metric on $[0,1]$ given by

$$
\rho(s, t)=\min \{|s-t|, 1-|s-t|\} .
$$

The main result of this article is the following theorem.

THEOREM 1. If a heuristic tour is built using a spacefilling curve $\psi$ that satisfies Assumptions 1-4, then there exists a strictly positive continuous function $\varphi$ of period 1 such that

$$
\lim _{n \rightarrow \infty} \frac{E S_{n}}{\varphi\left(\log _{p} n\right)}=1
$$

where $p$ is the integer of Assumptions 1 and 2.

3. Convergence of expectations. We first recall that a $\psi$ that satisfies Assumption 3 creates a natural correspondence between random variables with the uniform distribution on $[0,1]^{2}$ and $[0,1]$. We safely omit the routine proof.

LEMMA 1. Suppose that $X$ is a random variable that is uniformly distributed in $[0,1]^{2}$ and that $\psi:[0,1] \rightarrow[0,1]^{2}$ is a surjection. Let $\psi^{*}$ be a function that, for every $x \in[0,1]^{2}$, selects a preimage of $x$; that is, $\psi^{*}$ satisfies $\psi\left(\psi^{*}(x)\right)=x$. For $t$ defined by $t=\psi^{*}(X)$, we have the fact that $t$ is uniformly distributed in $[0,1]$, provided that the spacefilling curve $\psi$ satisfies the bimeasure preserving Assumption 3.

One key to the analysis of $S_{n}$ is that $E S_{n}$ has a tidy expression in terms of the independent (unordered) $t_{i}$ 's. If $d(s, t)$ is given by

$$
d(s, t)=\left\{\begin{array}{lll}
t-s & \text { if } & 0 \leq s \leq t \leq 1 \\
1-s+t & \text { if } & 0 \leq t \leq s \leq 1
\end{array}\right.
$$


then $d(s, t)$ describes the distance along the circle of unit circumference in the counterclockwise direction from $s$ to $t$, and we can write $S_{n}$ in the symmetrical form

$$
S_{n}=\sum_{i=1}^{n}\left\|\psi\left(t_{i}\right)-\psi\left(\left(t_{i}+\delta_{i}\right) \bmod 1\right)\right\|^{2},
$$

where

$$
\delta_{i}=\min _{t_{j} \in S_{i}} d\left(t_{i}, t_{j}\right)
$$

and $S_{i}=\left\{t_{1}, t_{2}, \ldots, t_{i-1}, t_{i+1}, \ldots, t_{n}\right\}$. Moreover, the variables $\delta_{i}$ and $t_{i}$ are independent, and for each $i$ the variable $\delta_{i}$ has probability density given by

$$
f(t)=(n-1)(1-t)^{n-2}
$$

so we can compute

$$
\begin{aligned}
E S_{n} & =E \sum_{i=1}^{n}\left\|\psi\left(t_{i}\right)-\psi\left(\left(t_{i}+\delta_{i}\right) \bmod 1\right)\right\|^{2} \\
& =n E\left\|\psi\left(t_{1}\right)-\psi\left(\left(t_{1}+\delta_{1}\right) \bmod 1\right)\right\|^{2} \\
& =n \int_{0}^{1} \int_{0}^{1}\|\psi(s)-\psi((s+t) \bmod 1)\|^{2}(n-1)(1-t)^{n-2} d s d t
\end{aligned}
$$

Finally, introducing

$$
m(t)=\int_{0}^{1}\|\psi(s)-\psi((s+t) \bmod 1)\|^{2} d s
$$

we end up with the following lemma.

LEMMA 2 (key representation). It holds that

$$
E S_{n}=n(n-1) \int_{0}^{1} m(t)(1-t)^{n-2} d t
$$

To use this representation, we must collect some properties of $m(t)$. From the definition of $m(\cdot)$ and the circular Lipschitz property (Assumption 4) of $\psi$, we immediately find a useful pointwise bound.

LEMMA 3. For $0 \leq t \leq 1$, we have

$$
m(t) \leq c_{\psi}^{2} \min (t, 1-t)
$$

where $c_{\psi}$ is the Lipschitz constant of Assumption 4.

To get to the deeper properties of $m(t)$, we first set $q=1 / p$ and define a sequence of related functions $\left\{f_{n}\right\}_{n \geq 0}$ on the increasing intervals $\left[0, p^{n} q\right]$ by

$$
f_{0}(t)=\int_{0}^{1-t}\|\psi(s+t)-\psi(s)\|^{2} d s, \quad 0 \leq t \leq q
$$

and

$$
f_{n}(t)=p^{n} f_{0}\left(\frac{t}{p^{n}}\right), \quad 0 \leq t \leq p^{n} q
$$


We also define a parallel sequence of functions $\left\{g_{n}\right\}_{n \geq 0}$ by

$$
g_{0}(t)=f_{0}(t)+c_{\psi}^{2} t^{2}, \quad 0 \leq t \leq q
$$

and

$$
g_{n}(t)=p^{n} g_{0}\left(\frac{t}{p^{n}}\right), \quad 0 \leq t \leq p^{n} q .
$$

The benefit of introducing $f_{n}$ and $g_{n}$ is that we can show that they share a common limit that offers insight into the behavior of $m(x)$. We first establish a monotonicity relationship.

LEMMA 4. There is a function $w(t)$ such that for all $n \geq 0$ we have

$$
f_{n}(t) \leq f_{n+1}(t) \leq w(t) \leq g_{n+1}(t) \leq g_{n}(t), \quad 0 \leq t \leq p^{n} q .
$$

Proof. For any $0 \leq x \leq q$, we see from the definition of $m(x)$ that

$$
m(x) \geq \sum_{i=1}^{p} \int_{(i-1) q}^{i q-x}\|\psi(s)-\psi(s+x)\|^{2} d s .
$$

Next, by the translation Assumption 2, by changing variables, and by the dilation Assumption 1, we obtain

$$
\begin{aligned}
\sum_{i=1}^{p} \int_{(i-1) q}^{i q-x}\|\psi(s)-\psi(s+x)\|^{2} d s & =p \int_{0}^{q-x}\|\psi(s)-\psi(s+x)\|^{2} d s \\
& =\int_{0}^{p q-p x}\left\|\psi\left(\frac{s}{p}\right)-\psi\left(\frac{s+p x}{p}\right)\right\|^{2} d s \\
& =\frac{1}{p} \int_{0}^{1-p x}\|\psi(s)-\psi(s+p x)\|^{2} d s \\
& =\frac{1}{p} m(p x) .
\end{aligned}
$$

By combining (9) and (8), we find the basic fact that

$$
m(x) \geq \frac{1}{p} m(p x)
$$

and from (10) the first inequality of (7) follows immediately.

Turning to the $g_{n}$, we first note by the definition of $g_{0}$ and identity (9) that for $0 \leq x \leq q$ we have

$$
\begin{aligned}
g_{0}(x) & =\left\{\sum_{i=1}^{p} \int_{(i-1) q}^{i q-x}\|\psi(s)-\psi(s+x)\|^{2} d s+\sum_{i=1}^{p-1} \int_{i q-x}^{i q}\|\psi(s)-\psi(s+x)\|^{2} d s\right\}+c_{\psi}^{2} x^{2} \\
& =\frac{1}{p} m(p x)+\sum_{i=1}^{p-1} \int_{i q-x}^{i q}\|\psi(s)-\psi(s+x)\|^{2} d s+\left\{c_{\psi}^{2} p x^{2}-c_{\psi}^{2}(p-1) x^{2}\right\} \\
& =\frac{1}{p} m(p x)+c_{\psi}^{2} p x^{2}+\sum_{1=1}^{p-1}\left\{\int_{i q-x}^{i q}\|\psi(s)-\psi(s+x)\|^{2} d s-c_{\psi} x^{2}\right\} .
\end{aligned}
$$


By the Lipschitz property of $\psi$, we have

$$
\int_{i q-x}^{i q}\|\psi(s)-\psi(s+x)\|^{2} d s-c_{\psi}^{2} x^{2} \leq 0
$$

so, after replacing $x$ by $x / p$, we have for $0 \leq x \leq 1$ that $g_{0}(x) \geq p g_{0}(x / p)$, and our inequality for $g_{0}$ immediately yields the last inequality of (7).

Local boundedness and monotonicity of the sequences $f_{n}(x)$ and $g_{n}(x)$ tell us the sequences have pointwise limits. The definition of $g_{n}$ further tells us that

$$
g_{n}(x)-f_{n}(x)=c_{\psi}^{2} x^{2} / p^{n}, \quad 0 \leq x \leq p^{n} q ;
$$

so, in fact, both $f_{n}(x)$ and $g_{n}(x)$ must have the same limit; moreover, if we denote this limit by $w(x)$, then by the first and last inequalities of (7) we have for $0 \leq x \leq p^{n} q$ that

$$
f_{n}(x) \leq w(x) \leq g_{n}(x)
$$

completing the proof of Lemma 4.

The next lemma shows how $w(x)$ approximates $m(x)$ and articulates a vital scaling property.

LEMMA 5. The function $w$ has the following properties:

(a) $w(t) \leq c_{\psi}^{2} t$ for $0 \leq t \leq 1$,

(b) For $0 \leq t \leq q=1 / p,|m(t)-w(t)| \leq c_{\psi}^{2} t^{2}$,

(c) $w(t)=p w(t / p)$.

Proof. By Lemma 3, we have $m(u) \leq c_{\psi}^{2} u$ for $0 \leq u \leq 1$; so, for $0 \leq x \leq p^{n} q$ we have

$$
f_{n}(x)=p^{n} m\left(x, p^{n}\right) \leq p^{n} c_{\psi}^{2} x / p^{n}=c_{\psi}^{2} x
$$

yielding (a). To show (b), we note that the case where $n=0$ in inequality ( 7 ) states that, for $0 \leq x \leq q$,

$$
m(x)=f_{0}(x) \leq m(x) \leq g_{0}(x)
$$

and, by the definition of $g_{0}$, we obtain

$$
|m(x)-w(x)| \leq\left|g_{0}(x)-f_{0}(x)\right|=c_{\psi}^{2} x^{2}, \quad 0 \leq x \leq q .
$$

All that remains is to establish (c). By Lemma 4 and the definition of $g_{0}$, we have

$$
\begin{aligned}
w(x / p) & =\lim _{n \rightarrow \infty} p^{n} m\left(\frac{x}{p \cdot p^{n}}\right) \\
& =\lim _{n \rightarrow \infty} \frac{1}{p}\left\{p^{n+1} m\left(\frac{x}{p^{n+1}}\right)\right\} \\
& =w(x) / p
\end{aligned}
$$

completing the proof of the lemma.

LEMMA 6 (first Laplace representation). As $n \rightarrow \infty$, we have

$$
E S_{n}=n^{2} \int_{0}^{1} m(t) e^{-n t} d t+O(1 / n)
$$


Proof. The difference between $E S_{n}$ and the integral of (15) is bounded by

$$
\int_{0}^{1} m(t)\left|n^{2} e^{-n t}-n(n-1)(1-t)^{n-2}\right| d t
$$

and, by Lemma $3, m(t)=O(t)$ as $t \rightarrow 0$; so easy estimates give the lemma.

We can modify this last representation slightly to obtain one with the form of a standard Laplace integral. First, we note that

$$
\begin{aligned}
E S_{n} & =n^{2} \int_{0}^{q} e^{-n t} m(t) d t+n^{2} \int_{q}^{1} e^{-n t} m(t) d t+O(1 / n) \\
& =n^{2} \int_{0}^{q} e^{-n t} w(t) d t+O(1 / n)
\end{aligned}
$$

since

$$
\int_{0}^{q} n^{2} e^{-n t}|m(t)-w(t)| d t \leq \int_{0}^{q} n^{2} e^{-n t} c_{\psi}^{2} t^{2} d t=O(1 / n)
$$

and

$$
n^{2} \int_{q}^{1} m(t) e^{-n t} d t \leq n^{2} \int_{q}^{1} c_{\psi}^{2} t e^{-n t} d t \leq c_{\psi} n^{2} e^{-n q} .
$$

Moreover, we have

$$
\int_{q}^{\infty} n^{2} w(t) e^{-n t} d t \leq n^{2} \int_{q}^{\infty} c_{\psi}^{2} t e^{-n t} d t=O\left(n^{2} e^{-q n}\right)
$$

so we have proved the following lemma.

LEMMA 7 (second Laplace representation). It holds that

$$
E S_{n}=n^{2} \int_{0}^{\infty} w(t) e^{-n t} d t+O(1 / n)
$$

All that remains is to show that the last integral is a periodic function of $\log _{p} n$. If we let $I(n)$ denote the value of the integral, divide the interval $[0, \infty)$ into subintervals $\left[p^{k} / n, p^{k+1} / n\right]$, where $-\infty<k<\infty$, and let $t=p^{k+u} / n$, then we obtain

$$
\begin{aligned}
I(n) & =n^{2} \sum_{k=-\infty}^{\infty} \int_{p^{k} / n}^{p^{k+1} / n} w(t) e^{-n t} d t \\
& =n \sum_{k=-\infty}^{\infty} \int_{0}^{1} w\left(p^{k+u} / n\right) p^{k+u} \exp \left(-p^{k+u}\right) \log p d u
\end{aligned}
$$

Using the key recursion relation of Lemma 5 , part (c), we have $w\left(p^{k+u} / n\right)=p^{k} w\left(p^{u} / n\right)$; so

$$
\begin{aligned}
I(n) & =\log p \int_{0}^{1} w\left(p^{u-\log _{p} n}\right) /\left(p^{u-\log _{p} n}\right)\left\{\sum_{k=-\infty}^{\infty} p^{2(k+u)} \exp \left(-p^{k+u}\right)\right\} d u \\
& =\log p \int_{0}^{1}\left\{w\left(p^{u-\log _{p} n}\right) /\left(p^{u-\log _{p} n}\right)\right\} l(u) d u
\end{aligned}
$$


where $l(u)$ is defined by

$$
l(u)=\sum_{k=-\infty}^{\infty} p^{2(k+u)} \exp \left(-p^{k+u}\right)
$$

Since the defining sum for $l$ converges uniformly on compact subsets of $[0, \infty)$, we see that $l$ is continuous. Furthermore, if we define a function $\varphi$ by

$$
\varphi(x)=\log p \int_{0}^{1}\left\{w\left(p^{-(x-u)}\right) /\left(p^{-(x-u)}\right)\right\} l(u) d u
$$

the continuity of $l$, the local boundedness of $w$, and the convolution form of (17) show that $\varphi$ is also continuous. Since $\varphi\left(\log _{p} n\right)=I(n)$, we can write Lemma 7 as

$$
E S_{n}=\varphi\left(\log _{p} n\right)+O(1 / n),
$$

and the proof of the theorem is completed once we establish the following lemma.

LEMMA 8. The function $\varphi$ is continuous, periodic of period 1 , and bounded away from zero.

Proof. We have already noted the continuity of $\varphi$, and periodicity is immediate from the recursion $w(p u)=p w(u)$ combined with the integral representation (17) of $\varphi$. The integral representation also gives $\varphi(x) \geq 0$ for all $x$.

By compactness, $\varphi$ will be bounded away from zero unless there is an $x_{0}$ such that $\varphi\left(x_{0}\right)=0$. However, for such an $x_{0}$, we would get from (17) that $w\left(p^{-\left(x_{0}-u\right)}\right)=0$ for all $0 \leq u \leq 1$. By the recursion $w(p u)=p w(u)$, we could then conclude that both $\varphi$ and $E S_{n}$ were identically zero. This contradiction establishes that $\varphi(x)$ is bounded away from zero. By $(18)$ and division by $\varphi\left(\log _{p} n\right)$, we find that

$$
\frac{E S_{n}}{\varphi\left(\log _{p} n\right)}=1+O(1 / n)
$$

which is more than we require to complete the proof of the theorem.

4. Beyond expectations. One finds no difficulty in extending Theorem 1 beyond the convergence of expectations to almost sure convergence. The first step is to obtain an understanding of $\operatorname{Var} S_{n}$, and this is easily approached though the use of martingales. For the martingale difference sequence defined by $d_{i}=E\left(S_{n} \mid \mathcal{F}_{i}\right)-$ $E\left(S_{n} \mid \mathcal{F}_{i-1}\right)$ where $\mathcal{F}_{i}=\sigma\left\{X_{1}, X_{2}, \ldots, X_{n}\right\}$, we have the representation

$$
S_{n}-E S_{n}=\sum_{i=1}^{n} d_{i}
$$

and, by the orthogonality of the martingale differences, we have

$$
\operatorname{Var} S_{n}=E\left|S_{n}-E S_{n}\right|^{2}=\sum_{i=1}^{n} E d_{i}^{2} .
$$

To help estimate $E d_{i}^{2}$, we introduce another collection of random variables $\left\{\tilde{X}_{i}, 1 \leq\right.$ $i \leq n\}$ that are assumed to be independent, uniformly distributed, and independent of the random variables $\left\{X_{i}, 1 \leq i \leq n\right\}$. We then let $S_{n}^{(i)}$ denote the sum of squares 
of the edges in the spacefilling heuristic tour of $\left\{X_{1}, X_{2}, \ldots, X_{i-1}, \tilde{X}_{i}, X_{i+1}, \ldots, X_{n}\right\}$ and note by $E\left(S_{n}^{(i)} \mid \mathcal{F}_{i}\right)=E\left(S_{n} \mid \mathcal{F}_{i-1}\right)$ that we have

$$
d_{i}=E\left(S_{n}-S_{n}^{(i)} \mid \mathcal{F}_{i}\right)
$$

The basic observation is that the tours associated with $S_{n}$ and $S_{n}^{(i)}$ differ by at most six edges. Furthermore, if we recall the oriented distance function $d(s, t)$ defined in $\S 3$ and specify integers $j_{1}$ and $j_{2}$ by the relations

$$
d\left(t_{j_{1}}, \tilde{t}_{i}\right)=\min _{j: j \neq i} d\left(t_{j}, \tilde{t_{i}}\right) \text { and } d\left(\tilde{t}_{i}, t_{j_{2}}\right)=\min _{j: j \neq i} d\left(\tilde{t}_{i}, t_{j}\right)
$$

then we obtain the spacefilling tour through $\left\{X_{1}, X_{2}, \ldots, X_{i-1}, \tilde{X}_{i}, X_{i+1}, \ldots, X_{n}\right\}$ by connecting $\tilde{X}_{i}$ into the spacefilling tour through $\left\{X_{1}, X_{2}, \ldots, X_{i-1}, X_{i+1}, \ldots, X_{n}\right\}$ by edges to $X_{j_{1}}$ and $X_{j_{2}}$ and removing the edge that connects $X_{j_{1}}$ and $X_{j_{2}}$. We are thus led to

$$
\begin{aligned}
S_{n}^{(i)}= & S\left(X_{1}, X_{2}, \ldots, X_{i-1}, X_{i+1}, \ldots, X_{n}\right)+\left\|\psi\left(t_{j_{1}}\right)-\psi\left(\tilde{t}_{i}\right)\right\|^{2} \\
& +\left\|\psi\left(\tilde{t_{i}}\right)-\psi\left(t_{j_{2}}\right)\right\|^{2}-\left\|\psi\left(t_{j_{1}}\right)-\psi\left(t_{j_{2}}\right)\right\|^{2} .
\end{aligned}
$$

Similarly, to build the spacefilling tour through $\left\{X_{1}, X_{2}, \ldots, X_{n}\right\}$ from the tour through $\left\{X_{1}, X_{2}, \ldots, X_{i-1}, X_{i+1}, \ldots, X_{n}\right\}$, we find $k_{1}$ and $k_{2}$ such that

$$
d\left(t_{k_{1}}, t_{i}\right)=\min _{k: k \neq i} d\left(t_{k}, t_{i}\right) \text { and } d\left(t_{i}, t_{k_{2}}\right)=\min _{k: k \neq i} d\left(t_{i}, t_{k}\right)
$$

from which we obtain

$$
\begin{aligned}
S_{n}= & S\left(X_{1}, X_{2}, \ldots, X_{i-1}, X_{i+1}, \ldots, X_{n}\right)+\left\|\psi\left(t_{k_{1}}\right)-\psi\left(t_{i}\right)\right\|^{2} \\
& +\left\|\psi\left(t_{i}\right)-\psi\left(t_{k_{2}}\right)\right\|^{2}-\left\|\psi\left(t_{k_{1}}\right)-\psi\left(t_{k_{2}}\right)\right\|^{2}
\end{aligned}
$$

The implied bound on $\left|S_{n}^{(i)}-S_{n}\right|$ is then

$$
\begin{aligned}
\left|S_{n}^{(i)}-S_{n}\right| \leq & \left\|\psi\left(t_{j_{1}}\right)-\psi\left(\tilde{t_{i}}\right)\right\|^{2}+\left\|\psi\left(\tilde{t_{i}}\right)-\psi\left(t_{j_{2}}\right)\right\|^{2} \\
& +\left\|\psi\left(t_{j_{1}}\right)-\psi\left(t_{j_{2}}\right)\right\|^{2}+\left\|\psi\left(t_{k_{1}}\right)-\psi\left(t_{i}\right)\right\|^{2} \\
& +\left\|\psi\left(t_{i}\right)-\psi\left(t_{k_{1}}\right)\right\|^{2}+\left\|\psi\left(t_{k_{1}}\right)-\psi\left(t_{k_{2}}\right)\right\|^{2}
\end{aligned}
$$

from which we find by Assumption 4 that $E\left|d_{i}\right|^{2} \leq E\left|S_{n}-S_{n}^{(i)}\right|^{2}$ is bounded by

$$
6 c_{\psi}^{2} E\left(\rho^{2}\left(t_{j_{1}}, \tilde{t}_{i}\right)+\rho^{2}\left(\tilde{t}_{i}, t_{j_{2}}\right)+\rho^{2}\left(t_{j_{1}}, t_{j_{2}}\right)+\rho^{2}\left(t_{k_{1}}, t_{i}\right)+\rho^{2}\left(t_{i}, t_{k_{2}}\right)+\rho^{2}\left(t_{k_{1}}, t_{k_{2}}\right)\right) .
$$

The computation of the expectations in (19) are now routine, given the known distribution of the gaps between $n$ (or $n-1$ ) points chosen on the unit circle. All of these expectations are $O\left(1 / n^{2}\right)$, and hence we have Lemma 9 .

LEMMA 9. For $n \rightarrow \infty$, the variance of the sum of squares of edges $S_{n}$ satisfies

$$
\operatorname{Var} S_{n}=O(1 / n)
$$

Now we are ready to prove the almost sure convergence of $S_{n}$. 
THEOREM 2. We have

$$
\lim _{n \rightarrow \infty} \frac{S_{n}}{\varphi\left(\log _{p} n\right)}=1 \quad \text { a.s. }
$$

Proof. The remaining steps follow a familiar pattern and will just be sketched. First, we consider a subsequence by letting $n_{i}=\left\lceil i \log ^{2} i\right\rceil$. By Chebyshev's inequality, for any $\epsilon>0$, we have

$$
\sum_{i=1}^{\infty} P\left(\left|S_{n_{i}}-E S_{n_{i}}\right|>\epsilon\right) \leq \epsilon^{-2} \sum_{i=2}^{\infty} \operatorname{Var} S_{n_{i}} \leq \epsilon^{-2} \sum_{i=2}^{\infty} i^{-1} \log ^{-2} i<\infty ;
$$

so, by the easy part of the Borel-Cantelli lemma, for $n \rightarrow \infty$, we have $\left(S_{n_{i}}-E S_{n_{i}}\right) \rightarrow 0$ with probability 1 . To stretch the convergence to the full sequence, we look at the largest difference $V_{i}$ of $S_{n}$ and $S_{n_{i}}$ as $n$ varies through the intervals $\left[n_{i}, n_{i+1}\right)$,

$$
V_{i}=\max _{n_{i} \leq n \leq n_{i+1}}\left|S_{n}-S_{n_{i}}\right| \text {. }
$$

For each $n$ such that $n_{i-1} \leq n \leq n_{i}$, the number of terms that occur in $S_{n}$ and not in $S_{n_{i}}$ or vice versa is at most $O\left(n_{i}-n_{i-1}\right)=O\left(\log ^{2} i\right)$. If $A$ denotes the set of all such terms, then $|A|=O\left(\log ^{4} i\right)$; and, if $L_{i}$ is the largest of these terms, then by Assumption 4 and the classical proof of the fact that the largest gap between $n$ points chosen at random from the circle is sharply concentrated around $n^{-1} \log n$, we can show that $E L_{i}^{2}=O\left(n_{i}^{-2} \log ^{2} n_{i}\right)$. Since $V_{i} \leq|A| L_{i}$, we see $E V_{i}^{2}=O\left(i^{-2} \log ^{10} i\right)$; so, again by the Borel-Cantelli lemma, we see that, for $n \rightarrow \infty$, we have $V_{i} \rightarrow 0$ with probability 1, which, in turn, completes the proof of the theorem.

5. Concluding remarks. We have established a striking property of the sum of squares of the heuristic tour provided by the spacefilling curve method. Of the questions that remain open, the most natural are perhaps those that seek a more detailed understanding of $\varphi$, the periodic function that figures in Theorem 1 . The (centered) supremum norm of $\varphi$ determines the strength of the oscillation of $E S_{n}$, and almost nothing is known about this norm. Simulations offer little insight because of the slow growth of $\log _{p} n$ and the difficulty of estimating $E S_{n}$. Still, calculations given in Platzman and Bartholdi [8] would suggest by analogy that the oscillation due to $\varphi$ is not large, perhaps only a few percent. This reinforces the difficulty of obtaining detailed information about $\varphi$ from simulations.

Another natural question addresses the possibility of obtaining sharp bounds on the tail probabilities $P\left(S_{n} \geq t\right)$. In view of the remarkable work of Rhee and Talagrand [9] on the Gaussian tail bound for the optimal length of the TSP tour length, we are tempted to suggest that $P\left(S_{n} \geq t\right) \leq A \exp \left(-B t^{2}\right)$ for all some $A$ and $B$ and all $t \geq 0$. Some of the structure that Rhee and Talagrand require (like the martingale used in $\S 4)$ is available in for $S_{n}$, but a detailed understanding of $P\left(S_{n} \geq t\right)$ seems to require additional insights, since, in particular, $S_{n}$ may have tails that are much lighter than those of the Gaussian distribution.

Perhaps the most compelling problems suggested by this work concern the behavior of the optimal tours rather than the heuristic cousins. First, if we let $S_{n}^{\text {TSP }}$ denote the sum of the squares of the edges in the (almost surely unique) shortest tour through a random sample of $n$ points chosen from the unit square, do we have

$$
\lim _{n \rightarrow \infty} E S_{n}^{\mathrm{TSP}}=C
$$


for some constant $C$ ?

Finally, there at least one compelling problem concerning the worst-case behavior of the sum of squared edges in an minimal length path. If $S$ is a finite subset of $[0,1]^{2}$ and $\operatorname{TSP}(S)$ denotes a path of minimal length through the points of $S$, we suspect that

$$
M_{n}=\max _{S:|S|=n} \sum_{e \in \operatorname{TSP}(S)}\|e\|^{2}
$$

is asymptotic to $c \log n$ as $n \rightarrow \infty$. By the results cited in the first section, we know $M_{n}=O(\log n)$ and $M_{n}=\Omega(\log n)$, but the present methods offer no serious progress toward a full asymptotic result. The periodicity that has been found for $E S_{n}$ shows that subtleties can emerge, though we need not expect them at every turn.

\section{REFERENCES}

[1] D. Aldous AND J. M. SteEle, Asymptotics for Euclidean minimal spanning trees on random points, Probab. Theory Rel. Fields, 92 (1992), pp. 247-258.

[2] J. BEARDWOOD, J. H. HALTON, AND J. M. HAMMERSLEY, The shortest path through many points, Proc. Cambridge Philos. Soc., 55 (1959), pp. 299-327.

[3] M. BERN AND D. EPSTEIN, Worst-case bounds for subadditive geometric graphs, Tech. Report, Xerox PARC, Palo Alto, CA, 1992.

[4] J. GaO AND J. M. STEele, General spacefilling curve heuristics and limit theory for the traveling salesman problem, J. Complexity, 10 (1994), to appear.

[5] D. HILbERT, Über die stetige Abbildung einer Linie auf ein Flächenstück, Math. Ann., 38 (1891), pp. 459-460.

[6] S. C. Milne, , Peano curves and smoothness of functions, Adv. Math., 35 (1980), pp. $129-157$.

[7] G. PEANo, Sur Une Courbe Qui Remplit Toute Une Aire Plane, Math. Ann., 36 (1890), pp. 157-160.

[8] L. K. PLATZMAN AND J. J. BARTHOLDI, Spacefilling curves and the planar traveling salesman problem, J. Assoc. Comput. Mach., 36 (1989), pp. 719-737.

[9] W. T. RhEE AND M. TAlagrand, A sharp deviation inequality for the stochastic traveling salesman problem, Ann. Probab., 17 (1989), pp. 1-8.

[10] T. L. SNYDER AND J. M. STEELE, A priori inequalities for the Euclidean traveling salesman problem, in Proc. 8th ACM Sympos. on Computational Geometry, Berlin, 1992, pp. 344349. 